EGU21-15776, updated on 08 Jun 2021

https://doi.org/10.5194/egusphere-egu21-15776

EGU General Assembly 2021

(c) Author(s) 2021. This work is distributed under

the Creative Commons Attribution 4.0 License.

\title{
Computer vision model for detecting block falls at the martian north polar region.
}

Oleksii Martynchuk, Lida Fanara, Ernst Hauber, Juergen Oberst, and Klaus Gwinner

Deutsches Zentrum für Luft- und Raumfahrt (DLR), Planetengeodesie, Germany (oleksii.martynchuk@dlr.de)

Dynamic changes of Martian north polar scarps present a valuable insight into the planet's natural climate cycles (Byrne, 2009; Head et al., 2003) ${ }^{1,2}$. Annual avalanches and block falls are amongst the most noticeable surface processes that can be directly linked with the extent of the latter dynamics (Fanara et al, 2020) ? $^{3}$. New remote sensing approaches based on machine learning allow us to make precise records of the aforementioned mass wasting activity by automatically extracting and analyzing bulk information obtained from satellite imagery. Previous studies have concluded that a Support Vector Machine (SVM) classifier trained using Histograms of Oriented Gradients (HOG) can be used to efficiently detect block falls, even against backgrounds with increased complexity (Fanara et al., 2020) ${ }^{4}$. We hypothesise that this pretrained model can now be utilized to generate an extended dataset of labelled image data, sufficient in size to opt for a deep learning approach. On top of improving the detection model we also attempt to address the image co-registration protocol. Prior research has suggested this to be a substantial bottleneck, which reduces the amounts of suitable images. We plan to overcome these limitations either by extending our model to include multi-sensor data, or by deploying improved methods designed for exclusively optical data (e.g. COSI-CORR software (Ayoub, Leprince and Avouac, 2017)5). The resulting algorithm should be a robust solution capable of improving on the already established baselines of $75.1 \%$ and $8.5 \%$ for TPR and FDR respectively (Fanara et al., 2020)4. The NPLD is our primary area of interest due to it's high levels of activity and good satellite image density, yet we also plan to apply our pipeline to different surface changes and Martian regions as well as on other celestial objects.

1. Head, J.W., Mustard, J.F., Kreslavsky, M.A., Milliken, R.E., Marchant, D.R., 2003. Recent ice ages on Mars. Nature 426, 797-802

2. Byrne, S., 2009. The polar deposits of Mars. Annu. Rev. Earth Planet. Sci. 37, 535-560.

3. Fanara, K. Gwinner, E. Hauber, J. Oberst, Present-day erosion rate of north polar scarps on Mars due to active mass wasting; Icarus,Volume 342, 2020; 113434, ISSN 0019-1035.

4. Fanara, K. Gwinner, E. Hauber, J. Oberst, Automated detection of block falls in the north polar region of Mars; Planetary and Space Science, Volume 180, 2020; 104733, ISSN 0032-0633. 
5. Ayoub, F.; Leprince, S.; Avouac, J.-P. User's Guide to COSI-CORR Co-registration of Optically Sensed Images and Correlation; California Institute of Technology: Pasadena, CA, USA, 2009; pp. $1-49$. 